\title{
In Vitro Activity of Iclaprim against Methicillin-Resistant Staphylococcus aureus Nonsusceptible to Daptomycin, Linezolid, or Vancomycin: A Pilot Study
}

\author{
David B. Huang, ${ }^{1,2}$ Stephen Hawser, ${ }^{3}$ Curtis G. Gemmell, ${ }^{4}$ and Daniel F. Sahm ${ }^{5}$ \\ ${ }^{1}$ Motif BioSciences, New York, NY, USA \\ ${ }^{2}$ Rutgers New Jersey Medical School, Trenton, NY, USA \\ ${ }^{3} I H M A$ Europe Sàrl, Route de I'Ile-au-Bois 1A, 1870 Monthey, Valais, Switzerland \\ ${ }^{4}$ Department of Bacteriology, University of Glasgow Medical School, Glasgow, UK \\ ${ }^{5}$ IHMA, Schaumburg, IL, USA \\ Correspondence should be addressed to David B. Huang; david.huang@motifbio.com
}

Received 31 July 2017; Revised 18 October 2017; Accepted 2 November 2017; Published 17 December 2017

Academic Editor: Paul-Louis Woerther

Copyright (C) 2017 David B. Huang et al. This is an open access article distributed under the Creative Commons Attribution License, which permits unrestricted use, distribution, and reproduction in any medium, provided the original work is properly cited.

\begin{abstract}
Iclaprim is a bacterial dihydrofolate reductase inhibitor in Phase 3 clinical development for the treatment of acute bacterial skin and skin structure infections and hospital-acquired bacterial pneumonia caused by Gram-positive bacteria. Daptomycin, linezolid, and vancomycin are commonly used antibiotics for these indications. With increased selective pressure to these antibiotics, outbreaks of bacterial resistance to these antibiotics have been reported. This in vitro pilot study evaluated the activity of iclaprim against methicillin-resistant Staphylococcus aureus (MRSA) isolates, which were also not susceptible to daptomycin, linezolid, or vancomycin. Iclaprim had an MIC $\leq 1 \mu \mathrm{g} / \mathrm{ml}$ to the majority of MRSA isolates that were nonsusceptible to daptomycin (5 of $7(71.4 \%))$, linezolid (26 of $26(100 \%))$, or vancomycin (19 of $28(66.7 \%))$. In the analysis of time-kill curves, iclaprim demonstrated $\geq 3 \log _{10}$ reduction in CFU/mL at $4-8$ hours for tested strains and isolates nonsusceptible to daptomycin, linezolid, or vancomycin. Together, these data support the use of iclaprim in serious infections caused by MRSA nonsusceptible to daptomycin, linezolid, or vancomycin.
\end{abstract}

\section{Background}

Iclaprim represents a diaminopyrimidine that inhibits bacterial dihydrofolate reductase of Gram-positive pathogens [1, 2]. Iclaprim exhibits potent in vitro activity against Grampositive pathogens associated with acute bacterial skin and skin structure infections (ABSSSIs) and nosocomial pneumonia including Staphylococcus aureus, Enterococcus spp., and Streptococcus spp. [1]. Iclaprim demonstrates rapid in vitro bactericidal activity in time-kill studies in human plasma [3]. Iclaprim is in Phase 3 clinical development for the treatment of ABSSSI and nosocomial pneumonia. Daptomycin, linezolid, and vancomycin are commonly used antibiotics for these indications (daptomycin is indicated for ABSSSI but not indicated for nosocomial pneumonia); however, increased selective pressure to these antibiotics has resulted in outbreaks of bacterial resistance to these antibiotics. Because of this emerging resistance, this current study was done to evaluate iclaprim's activity against MRSA isolates that were nonsusceptible to daptomycin, linezolid, or vancomycin.

\section{Materials and Methods}

Antibacterial susceptibility testing was conducted at the Department of Bacteriology, Glasgow Royal Infirmary, Glasgow, Scotland [4], and Eurofins Microbiology Laboratories on a range of MSSA and MRSA strains and isolates with varying susceptibilities to several recognized antistaphylococcal antibiotics. A total of 61 nonduplicative, nonconsecutive isolates of methicillin-resistant $S$. aureus (MRSA), which were nonsusceptible to daptomycin, linezolid, or vancomycin, were obtained from Eurofins repository or from the National 
TABLE 1: Iclaprim in vitro activity against MRSA isolates nonsusceptible to daptomycin, linezolid, or vancomycin.

\begin{tabular}{lccc}
\hline MRSA phenotype (number) & Iclaprim $\mathrm{MIC} \leq 1, \mu \mathrm{g} / \mathrm{mL}(\%)$ & Iclaprim $\mathrm{MIC}_{50} / \mathrm{MIC}_{90}(\mu \mathrm{g} / \mathrm{mL})$ & $\mathrm{MIC} \mathrm{range}(\mu \mathrm{g} / \mathrm{ml})$ \\
\hline Daptomycin nonsusceptible $(n=7)$ & $5 / 7(71.4)$ & $0.25 />8$ & $0.12->8$ \\
Linezolid nonsusceptible $(n=26)$ & $26 / 26(100.0)$ & $0.06 / 0.25$ & $0.03-1$ \\
Vancomycin intermediate $(n=23)$ & $16 / 23(69.6)$ & $0.25 />8$ & $0.25->8$ \\
Vancomycin resistant $(n=5)$ & $3 / 5(60.0)$ & $0.5 />8$ & $0.25->8$ \\
\hline
\end{tabular}

Institutes of Health Network on Antimicrobial Resistance to Staphylococcus aureus (NARSA) repository.

Clinical isolates were identified by the submitting laboratories, and identifications were confirmed centrally at Eurofins using the Bruker matrix-assisted laser desorption ionizationtime of flight mass spectrometry (MALDI-TOF) biotyper. Susceptibility testing and minimal inhibitory concentration (MIC) interpretations were performed according to broth microdilution protocols. S. aureus breakpoints for daptomycin, linezolid, and vancomycin are $\leq 1, \leq 4$, and $\leq 2 \mu \mathrm{g} / \mathrm{mL}$ $(4-8 \mu \mathrm{g} / \mathrm{mL}$ were classified as vancomycin-intermediate $S$. aureus and $\geq 16$ were classified as vancomycin-resistant $S$. aureus), respectively. To date, there are no published clinical breakpoints for iclaprim. However, based on a number of factors (e.g., MRSA distribution of MICs, assessment of the pharmacokinetics/pharmacodynamics of iclaprim, and the study of the clinical outcomes of MRSA infections when iclaprim was used in Phase 2 and 3 studies) outlined in the CLSI M23 guideline, an iclaprim MIC $\leq 1 \mu \mathrm{g} / \mathrm{mL}$ for $S$. aureus, including MRSA, has been proposed.

MRSA isolates were tested in cation-adjusted MuellerHinton broth (CA-MHB). Quality control and interpretation of results were performed in accordance with CLSI M100-S25 methods [5]. QC ranges for iclaprim were those approved by CLSI and published in M100-S25 [4]. Iclaprim and comparator antibiotic MIC results were within the CLSI published ranges against S. aureus ATCC 29213. Isolates were tested with MIC panels (Thermo Fisher Scientific, Cleveland, Ohio, USA) of comparator antibiotics (trimethoprim, trimethoprim-sulfamethoxazole, ceftriaxone, erythromycin, levofloxacin, oxacillin, meropenem, tetracycline, tigecycline, vancomycin, linezolid, and daptomycin).

The analysis of time-kill curves was performed by exposing $10^{5}-10^{6} \mathrm{CFU} / \mathrm{mL}$ of each MRSA isolate or strain to iclaprim, daptomycin, linezolid, or vancomycin at 2,4 , and $8 x$ MICs. Bactericidal activity was defined as a $\geq 3 \log _{10}$ reduction in $\mathrm{CFU} / \mathrm{mL}$ after 24 hours of incubation.

\section{Results}

The $\mathrm{MIC}_{50}$ and $\mathrm{MIC}_{90}$ values for the MRSA nonsusceptible to daptomycin, nonsusceptible to linezolid, vancomycinintermediate, and vancomycin-resistant were $>1 />1,8 />8$, $4 / 8$, and $>32 />32 \mu \mathrm{g} / \mathrm{mL}$, respectively. Among the seven MRSA isolates nonsusceptible to daptomycin (all seven had an MIC > 1 to daptomycin), four, two, and one had a vancomycin MIC of 4,8 , and $2 \mu \mathrm{g} / \mathrm{mL}$, respectively.

Table 1 shows that iclaprim exhibited potent activity against the majority of the 61 MRSA isolates that were nonsusceptible to daptomycin, linezolid, or vancomycin
$\left(\mathrm{MIC}_{50} 0.25 \mu \mathrm{g} / \mathrm{mL}\right)$. In the Glasgow study, all strains and isolates of MRSA and MSSA had an iclaprim MIC $\leq 1 \mu \mathrm{g} / \mathrm{mL}$. Iclaprim notably exhibited $100 \%$ activity against MRSA isolates $(n=26)$ that were nonsusceptible to linezolid. A total of 9 $(15.2 \%)$ isolates had reduced susceptibility to iclaprim with MICs $>8 \mu \mathrm{g} / \mathrm{mL}$ (Table 1). These isolates were not clustered in time of isolate collection, infection type, and/or geographic region. Figure 1 shows representative time-kill curves of iclaprim $(2 \mathrm{x}, 4 \mathrm{x}$, and $8 \mathrm{x}$ MICs for all antibiotics), which exhibited bactericidal activity at $4-8$ hours against MRSA strains and isolates nonsusceptible to daptomycin, linezolid, or vancomycin. As expected, representative time-kill curves of daptomycin exhibited no activity against MRSA strains and isolates nonsusceptible to daptomycin, linezolid exhibited no activity against MRSA strains and isolates nonsusceptible to linezolid, and vancomycin exhibited no activity against MRSA strains and isolates nonsusceptible to vancomycin.

\section{Discussion}

This report shows that iclaprim, without a synergistic combination of a sulfonamide, was highly active and rapidly bactericidal against a collection of 61 MRSA clinical isolates with nonsusceptible phenotypes to daptomycin, linezolid, or vancomycin. The $\mathrm{MIC}_{50}$ value of $0.25 \mu \mathrm{g} / \mathrm{mL}$ for MRSA documented in this study was consistent with $\mathrm{MIC}_{50}$ values in two previous surveillance reports for 5937 Gram-positive isolates, including MRSA, beta-hemolytic streptococci (most commonly Streptococcus pyogenes and S. agalactiae), and S. pneumoniae [1]. These isolates were collected from patients in the US and EU with skin and soft tissue, blood stream, and respiratory clinical specimens.

Based on MIC distributions of MRSA, assessment of the pharmacokinetics and pharmacodynamics of iclaprim, and the study of the clinical outcomes of MRSA infections when iclaprim was used in Phase 2 and 3 studies, an iclaprim $\mathrm{MIC} \leq 1 \mu \mathrm{g} / \mathrm{mL}$ for $S$. aureus, including MRSA, has been proposed as the breakpoint for nonsusceptibility. The $80 \mathrm{mg}$ fixed dose is based on prior animal models of infection studies, which suggest that the pharmacokinetic and pharmacodynamics (PK/PD) drivers, which best correlated with efficacy, were the area under the curve from 0 to 24 hours at steady state $\left(\mathrm{AUC}_{0-24 \mathrm{~h}, \mathrm{ss}}\right), \mathrm{AUC} / \mathrm{minimum}$ inhibitory concentration (MIC), and time above the MIC during the dosing interval ( $\mathrm{T}>\mathrm{MIC}$ ). In addition, using $\mathrm{PK}$ data collected from 470 patients from a Phase 3-complicated skin and skin infection (cSSSI) trials (ASSIST-1 and 2), population PK modeling, and Monte Carlo simulation identified that the fixed iclaprim $80 \mathrm{mg}$ dosage regimen optimally maximized $\mathrm{AUC}_{0-24 \mathrm{~h}, \mathrm{ss}}$, AUC/MIC, and T $>$ MIC while minimizing 
(A)

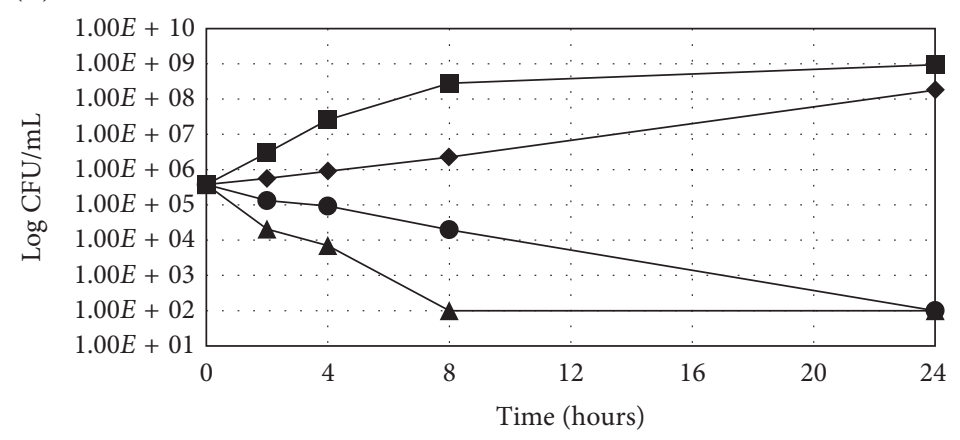

$$
\begin{aligned}
& - \text { Growth control } \\
& - \text { Iclaprim } \\
& - \text { - Vancomycin } \\
& - \text { - Linezolid }
\end{aligned}
$$

(B)

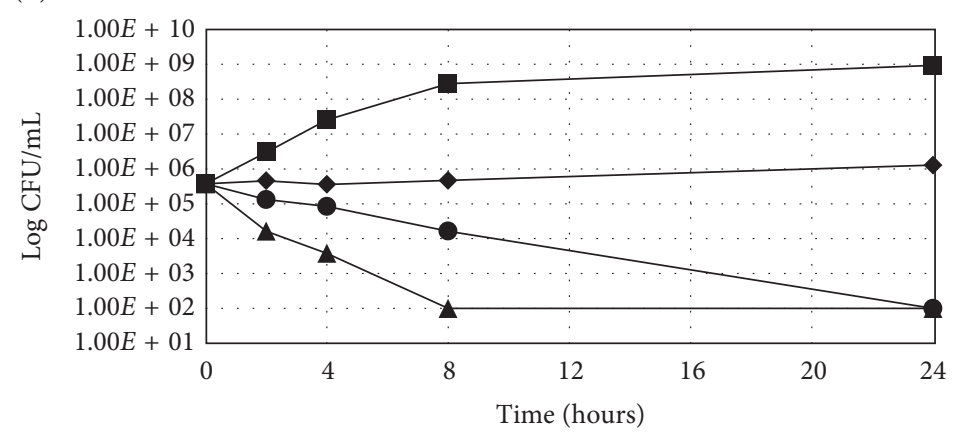

$$
\begin{aligned}
& - \text { Growth control } \\
& - \text { Iclaprim } \\
& - \text { Vancomycin } \\
& - \text { Linezolid }
\end{aligned}
$$

(C)

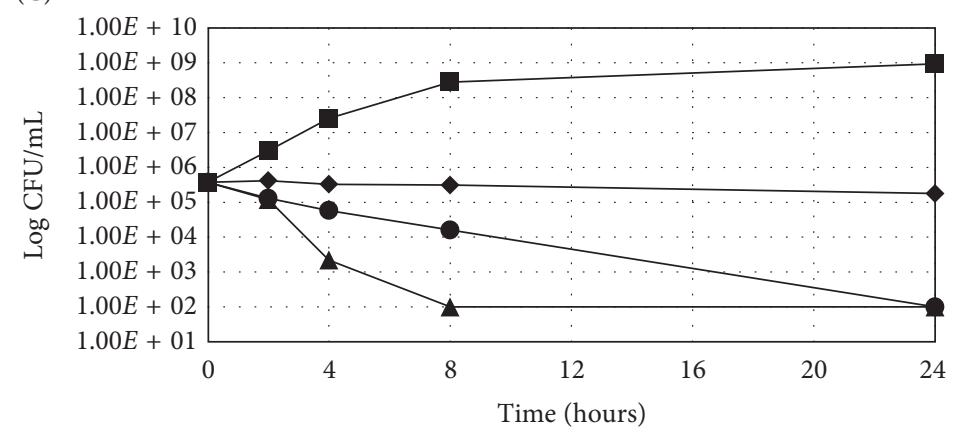

Growth control

— Iclaprim

- Vancomycin

$\checkmark$ Linezolid 
(A)

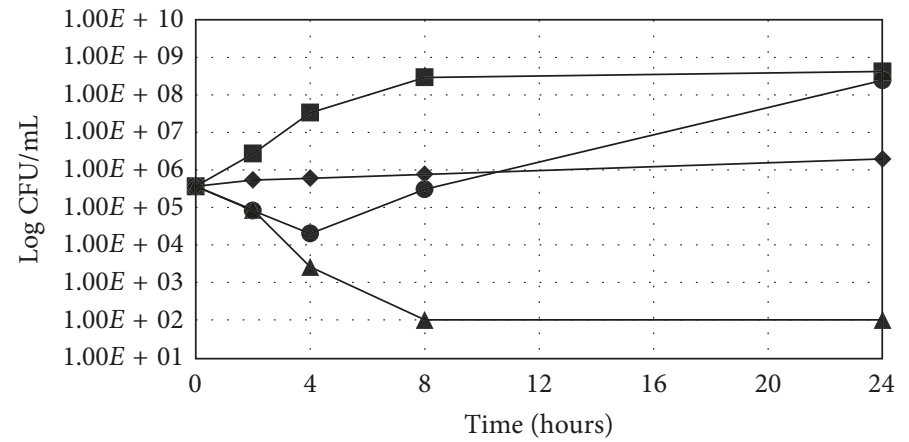

- Growth control

\ Iclaprim

- Vancomycin

$\multimap$ Linezolid

(B)

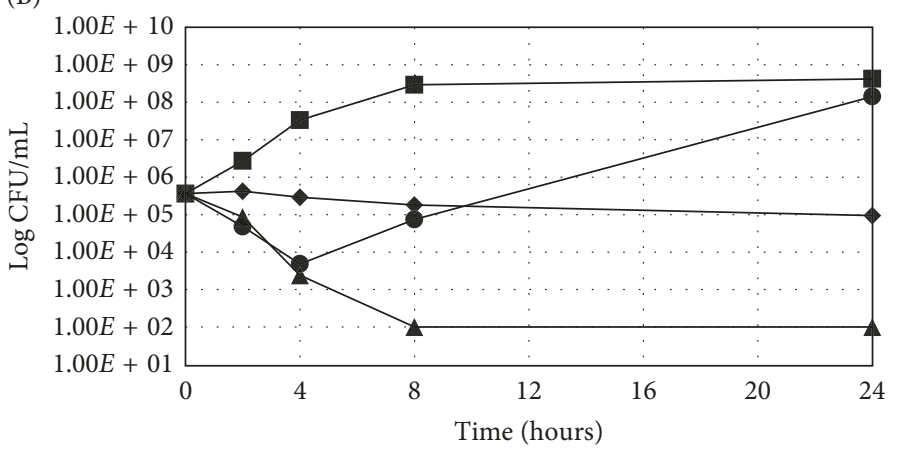

$-\mathrm{E}$ - Growth control
- Iclaprim
- - Vancomycin
- - Linezolid

(C)

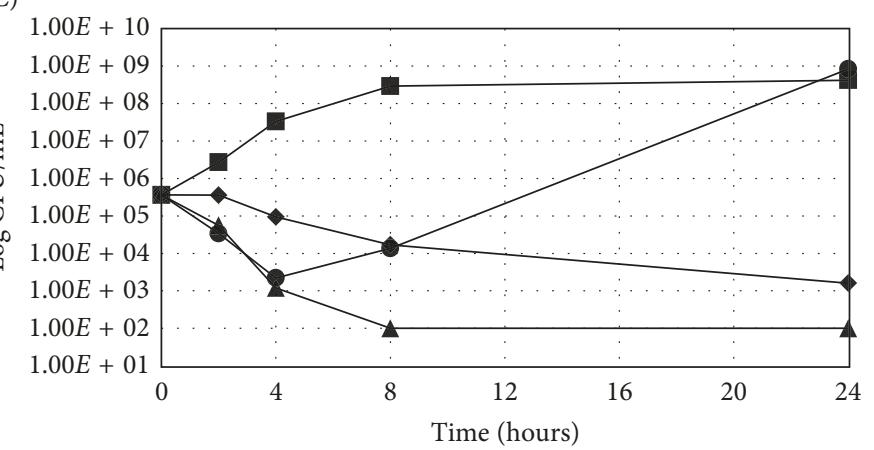

를- Growth control

$\triangle$ Iclaprim

- Vancomycin

$\multimap$ Linezolid 


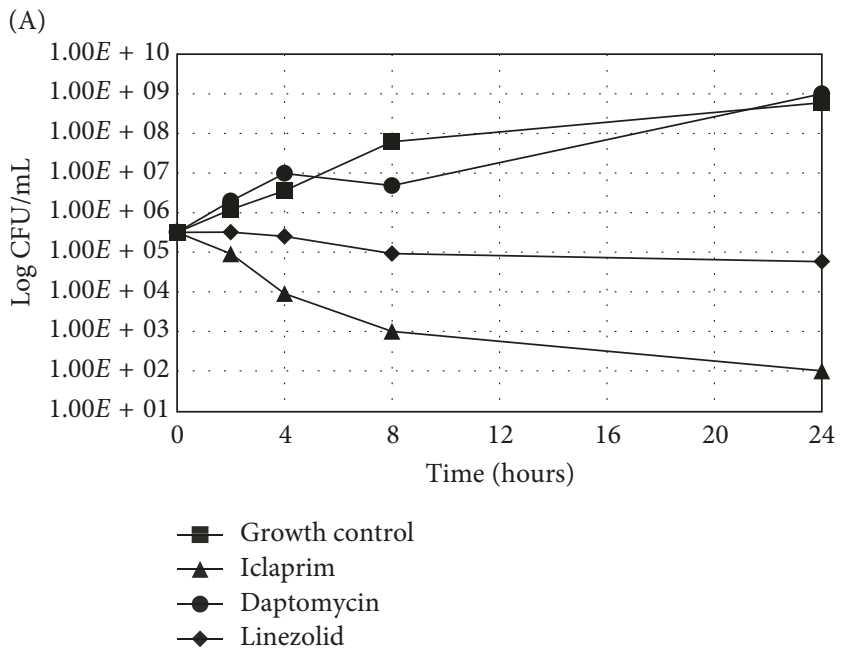

(B)
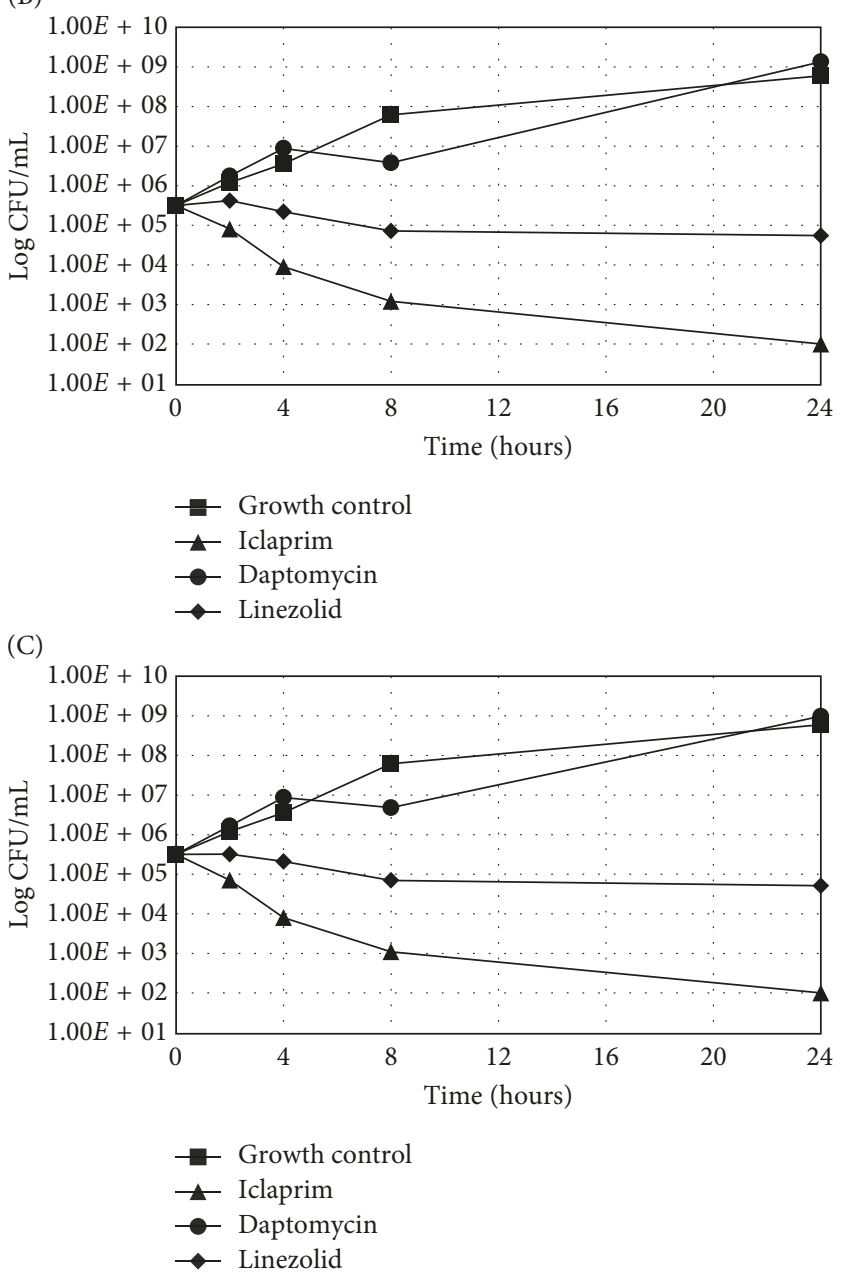

(c)

FIgURE 1: Iclaprim time-kill curves against MRSA isolates nonsusceptible to linezolid, resistant to vancomycin, and nonsusceptible to daptomycin, 2x, 4x, and 8x MICs were used for all antibiotics. (a) MRSA, linezolid nonsusceptible strain (MIC $\geq 8 \mu \mathrm{g} / \mathrm{mL}$ ), ATCC 986537, NRS271. (A) $2 \mathrm{x}$ MIC. (B) 4x MIC. (C) 8x MIC. Iclaprim showed significantly lower CFU at $2 \mathrm{~h}, 4 \mathrm{~h}, 8 \mathrm{~h}$, and $24 \mathrm{~h}$ compared to control, vancomycin, and linezolid $(P<0.01$; one-way ANOVA with Tukey's post hoc test). (b) MRSA, vancomycin-resistant strain $(\mathrm{MIC} \geq 32 \mu \mathrm{g} / \mathrm{mL}), \mathrm{ATCC} 1409053$, vanA positive. (A) 2x MIC. (B) 4x MIC. (C) 8x MIC. Iclaprim showed significantly lower CFU at $4 \mathrm{~h}, 8 \mathrm{~h}$, and $24 \mathrm{~h}$ compared to control, vancomycin, and linezolid $(P<0.01$; one-way ANOVA with Tukey's post hoc test). (c) MRSA, daptomycin-resistant strain (MIC $\geq 4 \mu \mathrm{g} / \mathrm{mL}$ ) (clinical isolate). (A) 2x MICs. (B) 4x MIC. (C) 8x MIC. Iclaprim showed significantly lower CFU at $4 \mathrm{~h}, 8 \mathrm{~h}$, and $24 \mathrm{~h}$ compared to control, daptomycin, and linezolid $(P<0.01$; one-way ANOVA with Tukey's post hoc test). 
the probability of a $C_{\text {max,ss }}$ to $\geq 800 \mathrm{ng} / \mathrm{mL}$, a concentration associated with dose-limiting toxicity [6]. Based on PK/PD analyses, iclaprim $80 \mathrm{mg}$ administered over two hours every 12 hours adequately covers $S$. aureus clinical isolates with an iclaprim MIC $\leq 1 \mu \mathrm{g} / \mathrm{mL}$; therefore, this dose was selected as the dosing scheme for ongoing Phase 3 clinical trials.

A limitation of this study is the small numbers of daptomycin and linezolid nonsusceptible and vancomycinresistant MRSA strains to arrive at conclusive activity of iclaprim against these types of strains and dose selection justification for clinical trials, which robust in vitro data are necessary. However, these data suggest that larger studies are warranted in examining iclaprim's activity against daptomycin and linezolid nonsusceptible and vancomycin-resistant MRSA. The findings of reduced daptomycin susceptibility and reduced vancomycin susceptibility and resistance have been reported in S. aureus. Daptomycin and vancomycin crossresistance is believed to be related to the physical barrier of a thickened cell wall of MRSA against the penetration of daptomycin and vancomycin molecules [7, 8]. A possible reason as to why iclaprim had reduced activity against such isolates may relate to the mechanism of action of iclaprim, which interferes with folate metabolism in the bacterial cell by competitively blocking the biosynthesis of tetrahydrofolate. This product acts as a carrier of one-carbon fragments and is necessary for the ultimate synthesis of DNA, RNA, and bacterial cell wall proteins. As vancomycin-resistant strains are already altered in terms of cell wall targets, it is likely that some products of folate metabolism are less important [9].

The results of this in vitro study suggest that iclaprim may be a useful treatment option for infections caused by MRSA, including those with nonsusceptible phenotypes to daptomycin, linezolid, or vancomycin. Daptomycin, linezolid, and vancomycin are antibiotics that are FDA approved, and the Infectious Diseases Society of America guidelines list these antibiotics as treatment options for skin and skin structure infections (SSSIs) caused by Grampositive pathogens [10]. New therapeutic options are needed, especially because of reported nonsusceptibility of Grampositive bacteria to daptomycin, linezolid, and vancomycin and its associated poor outcomes, increased length of stay, healthcare costs, and overall morbidity [11-14].

\section{Conclusion}

In conclusion, the results from this pilot in vitro study show the potent and rapid bactericidal activity of iclaprim against clinical MRSA isolates, including those with nonsusceptible phenotypes to daptomycin, linezolid, or vancomycin. Continued surveillance is warranted to track the continued potency of iclaprim, as well as MRSA isolates nonsusceptible to daptomycin, linezolid, and vancomycin and to detect any potential emergence of resistance.

\section{Conflicts of Interest}

David B. Huang is an employee of Motif BioSciences. Stephen Hawser was a former employee of Arpida. Daniel F. Sahm was a former employee of Eurofins.

\section{Acknowledgments}

This study was funded by Motif BioSciences.

\section{References}

[1] H. S. Sader, T. R. Fritsche, and R. N. Jones, "Potency and bactericidal activity of iclaprim against recent clinical grampositive isolates," Antimicrobial Agents and Chemotherapy, vol. 53, no. 5, pp. 2171-2175, 2009.

[2] P. Schneider, S. Hawser, and K. Islam, "Iclaprim, a novel diaminopyrimidine with potent activity on trimethoprim sensitive and resistant bacteria," Bioorganic and Medicinal Chemistry Letters, vol. 13, no. 23, pp. 4217-4221, 2003.

[3] H. Laue, T. Valensise, A. Seguin, S. Lociuro, K. Islam, and S. Hawser, "In vitro bactericidal activity of iclaprim in human plasma," Antimicrobial Agents and Chemotherapy, vol. 53, no. 10, pp. 4542-4544, 2009.

[4] C. G. Gemmell and G. Middlemas, "AR-100, a novel diaminopyrimidine: activity against various clinical isolates of gram-positive and gram-negative bacteria," in Proceedings of 42nd Interscience Conference on Antimicrobial Agents and Chemotherapy (ICAAC), San Diego, CA, USA, September 2002.

[5] CLSI M100-S25, Performance Standards for Antimicrobial Susceptibility Testing: 25th Informational Supplement, Clinical and Laboratory Standards Institute, Wayne, PA, USA, 2015.

[6] D. B. Huang and T. L. Lodise, "Use of pharmacokinetic/ pharmacodynamics (PK/PD) analyses to determine the optimal fixed dosing regimen of iclaprim for Phase III ABSSSI clinical trials," in Proceedings of IDWeek 2016, San Diego, CA, USA, October 2016.

[7] L. Z Cui, E. Tominaga, H. Neoh, and K. Hiramatsu, "Correlation between reduced daptomycin susceptibility and vancomycin resistance in vancomycin-intermediate Staphylococcus aureus," Antimicrobial Agents and Chemotherapy, vol. 50, no. 3, pp. 1079-1082, 2006.

[8] L. Z. Cui, X. Ma, K. Sato et al., "Cell wall thickening is a common feature of vancomycin resistance in Staphylococcus aureus," Journal of Clinical Microbiology, vol. 41, no. 1, pp. 5-14, 2003.

[9] H. C. Neu and T. D. Gootz, "Antimicrobial chemotherapy," in Medical Microbiology, S. Baron, Ed., University of Texas Medical Branch at Galveston, Galveston, TX, USA, 4th edition, 1996.

[10] D. L. Stevens, A. L. Bisno, H. F. Chambers et al., "Practice guidelines for the diagnosis and management of skin and soft tissue infections: 2014 update by the Infectious Diseases Society of America," Clinical Infectious Diseases, vol. 59, no. 2, pp. 147-159, 2014.

[11] M. Bassetti, E. Righi, and A. Carnelutti, "New therapeutic options for skin and soft tissue infections," Current Opinion in Infectious Diseases, vol. 29, no. 2, pp. 99-108, 2016.

[12] J. Edelsberg, A. Berger, D. J. Weber, R. Mallick, A. Kuznik, and G. Oster, "Clinical and economic consequences of failure of initial antibiotic therapy for hospitalized patients with complicated skin and skin-structure infections," Infection Control and Hospital Epidemiology, vol. 29, no. 2, pp. 160-169, 2008.

[13] K. J. Eagye, A. Kim, S. Laohavaleeson, J. L. Kuti, and D. P. Nicolau, "Surgical site infections: does inadequate antibiotic therapy affect patient outcomes?," Surgical Infections, vol. 10, no. 4, pp. 323-331, 2009.

[14] E. Nannini, B. E. Murray, and C. A. Arias, "Resistance or decreased susceptibility to glycopeptides, daptomycin, and linezolid in methicillin-resistant Staphylococcus aureus," Current Opinion in Pharmacology, vol. 10, no. 5, pp. 516-521, 2010. 


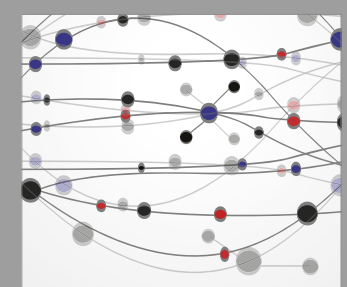

The Scientific World Journal
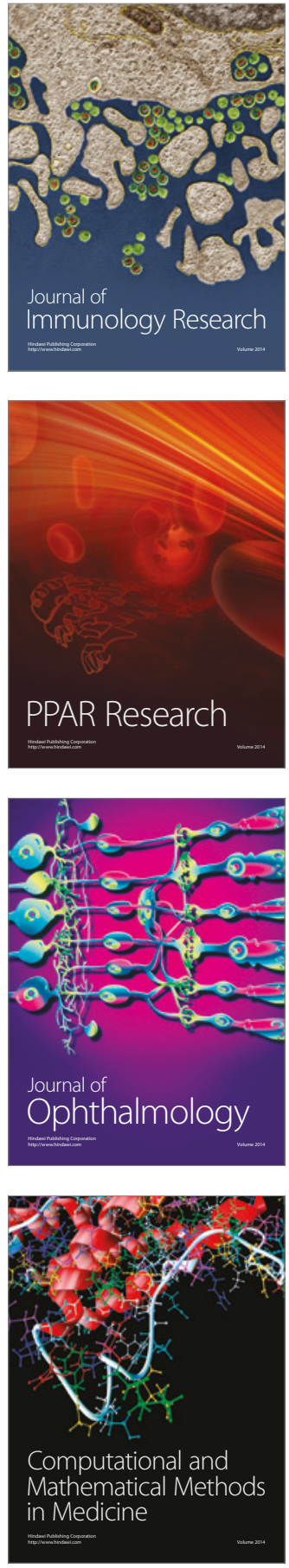

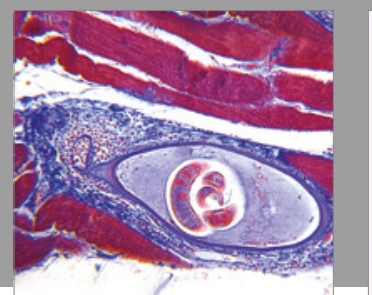

Gastroenterology Research and Practice
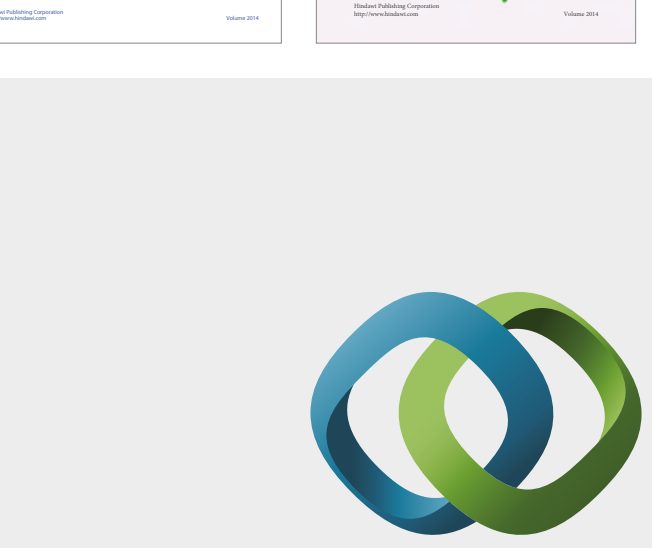

\section{Hindawi}

Submit your manuscripts at

https://www.hindawi.com
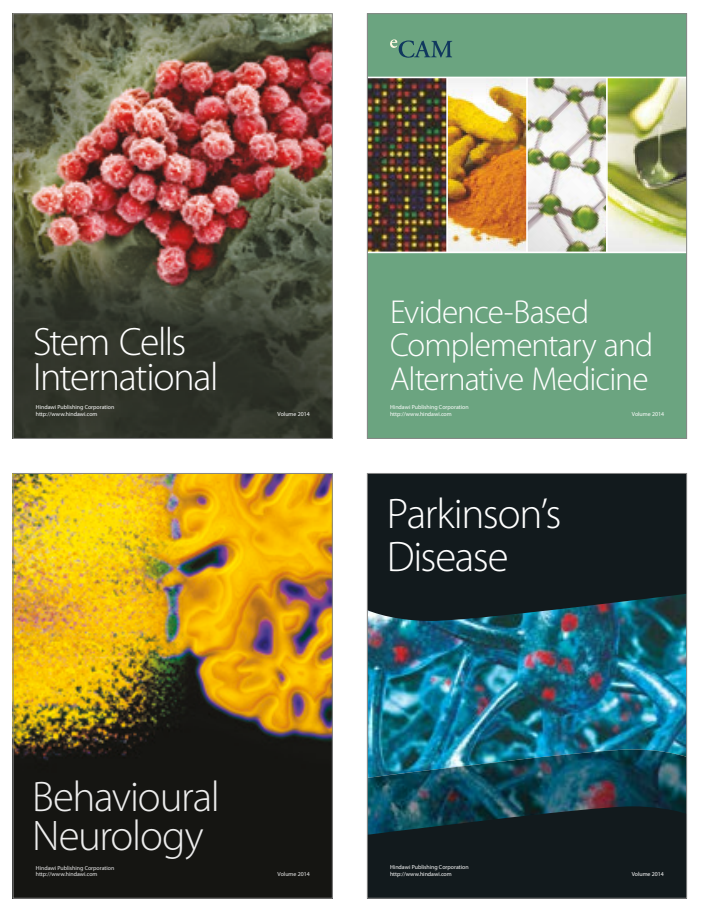
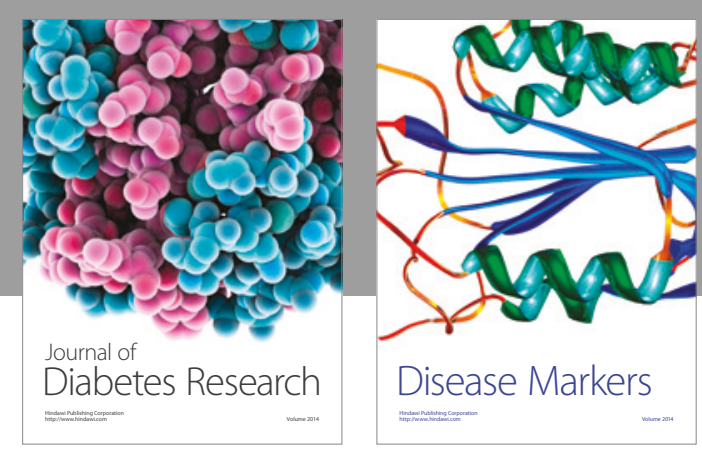

Disease Markers
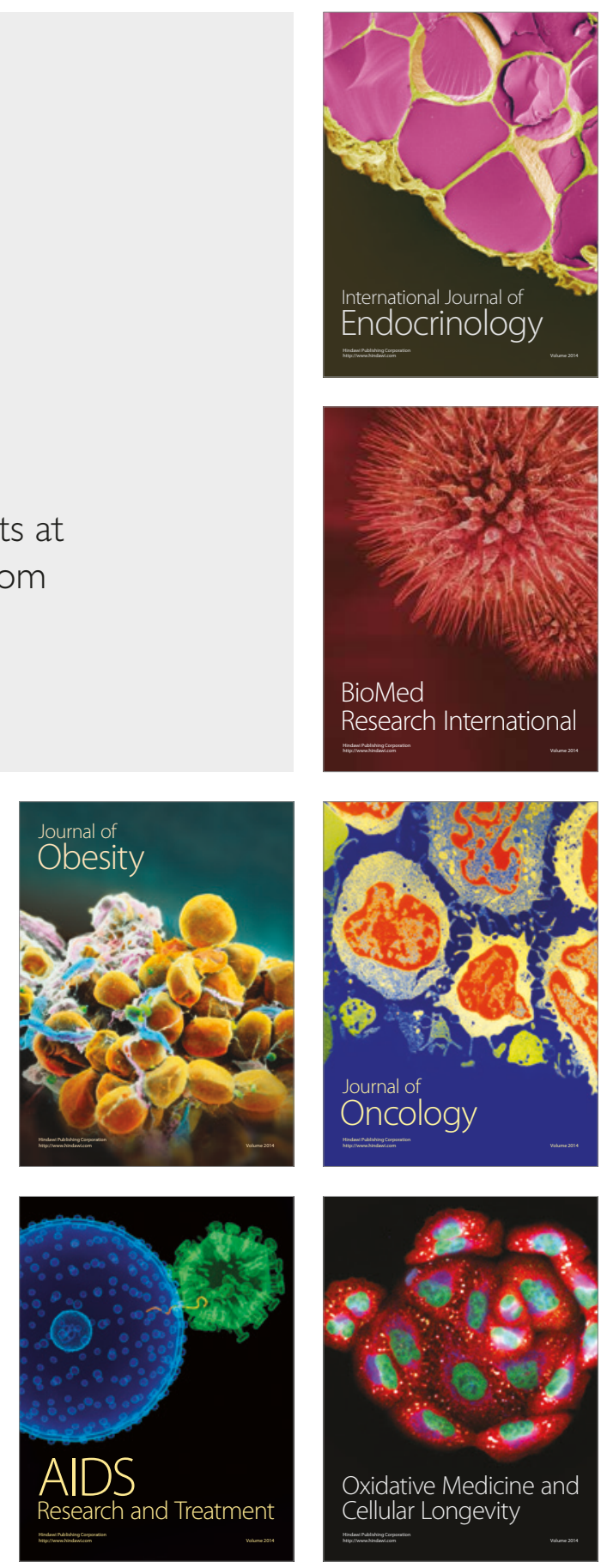